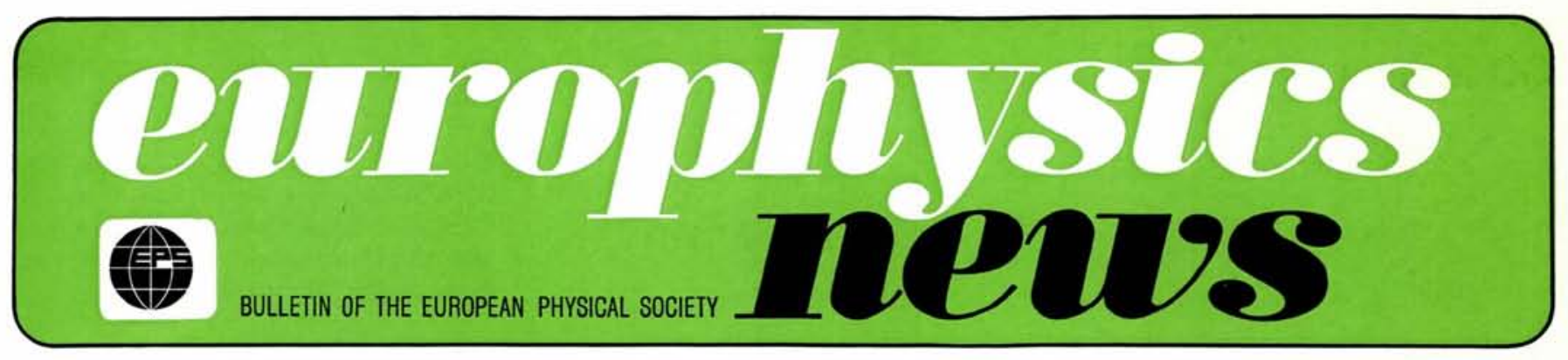

\title{
Optical Data Processing
}

\section{R. Van Geen, Brussels \\ (Rector of the Free University)}

\author{
(Based on a lecture given to Council members at Brugge, in March)
}

Opical data processing has become one of the most promising developments of modern optics, offering the possibilities of a new and powerful tool which will have an important impact on the structure of to-morrows's computers ${ }^{2-8}$ ).

The basic idea of optical data processing and retrieval or, more simply, optical computers can be summarized by the block diagram of Fig. 1. Taking the numbered elements shown in this figure, we can add the following:

1. The laser has become the appropriate source of coherent (in space and in time), monochromatic, stable light. It has made it possible, technologically speaking, to exploit the inherent potentialities of "Fourier Optics", holography included.

2. The input devices are, in the simplest cases, photographic plates which have the appropriate transmission function. More generally they are electro-optical (Kerr), magneto-optical or acoustical-optical devices, fed with the appropriate electrical signals ${ }^{3}$ ).

4. The optical transformer, transforms the light beam in the appropriate way, using various kinds of optical devices such as lenses, filters, diffracting masks, holograms, etc.
6. The output transducer may be either static, such as a photographic plate or a photochromic material or, dynamic, when it will be some form of photocell.

The advantage of the optical computer, in this form, as against the digital computer lies in its capability to treat in a time, characterized by the propagation time of light through the transformer, an enormous amount of data, in parallel processing, continuous and global, and not in a discrete and sequential way. Optical systems have two degrees of freedom for handling data ; these are the spatial coordinates in the wave plane. Electronics systems have only one independent variable, namely time. Nevertheless, the precision of optical systems remains for the moment relatively low, $1 \%$, compared to digital computers in most applications.

$U\left(x_{2}, y_{2}\right)=\mathrm{A} \exp \left[i k\left(1-d_{0} / f\right)\left(x_{2}{ }^{2}+y_{2}{ }^{2}\right) / 2 f\right] \times{ }_{\text {wave plane }} \mathrm{t}_{1}\left(x_{1}, y_{1}\right) \exp \left[-2 \pi i\left(x_{1} x_{2}+y_{1} y_{2}\right) / \lambda f\right] d x, d y_{1}$

\section{Basic Theory}

Optical processors rely on the basic theorem of image formation of $E$. Abbe, which connects Fourier transforms and the imaging properties of lenses (Fig. 2).

The amplitude and phase at coordinate $\left(x_{2} y_{2}\right)$ in the back focal plane are related to the Fourier transform of $t_{1}\left(x_{1}, y_{1}\right)$, in other words to the amplitude and phase of the object spec-

The amplitude distribution and in the back focal plane $\left(\begin{array}{ll}x_{2} & y_{2}\end{array}\right)$, can be easily obtained in the following way:

1. The beam of uniform amplitude is modulated by the amplitude filter, with transmission function $t,\left(x, y_{1}\right)$.

2. The propagation from the object to the plane in front of the lens is computed with the aid of the Kirchhoff-Fresnel scalar theory.

3. The lens, acting as a phase transformer, modulates the beam.

4. Propagation theory again allows the energy distribution in the back focal plane, at distance $t$ to be computed.

The result of the calculation gives the amplitude distribution $U$ in the plane $x_{2} y_{2}$ :

Fig. 1

1 Spatially and temporally coherent monochromatic radiation (laser)

2 Input transducer, which modulates the amplitude and/or phase of the light

3 Modulated radiation

4 Optical computer (lenses, filters, diffraction devices, holograms)

5 Transformed radiation

6 Output transducer, which records the computed results
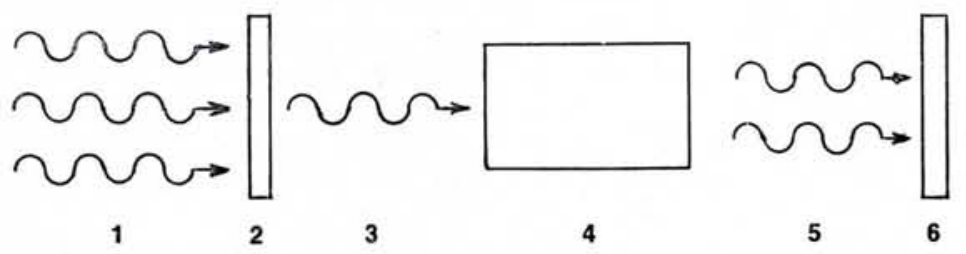

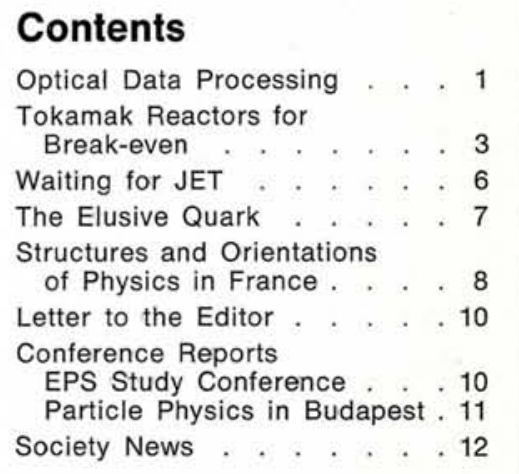


Fig. 2 Simple lens system underlying optical processing systems.

trum. A special case of particular importance is when $d_{0}=t$. The phase factor then disappears and one obtains directly the Fourier transform in the back focal plane.

\section{Optical Computers, Type 1}

In the first type of optical computer used for spectrum analysis, the optical system is reduced to a combination of lenses. It will be evident from the preceding paragraph that an optical arrangement similar to that shown in Fig. 2 can make up a computer which will perform very easily and very quickly the Fourier transform (more generally any integral transform) of a function of two independent variables.

Many industrial spectrum analyzers of this type have been built, some of which have been extended to multichannel spectrum analysis. They perform a spatial Fourier analysis of a given spatial distribution, similar to the classical Fourier analysis of timedependent periodic functions. Applications are numerous and can be found in such diverse fields as antenna pattern analysis, signal spectrum analysis in television, syntheticaperture techniques in radar, etc. ${ }^{3}$ ).

\section{Optical Computers, Type 2}

For the second type of optical computer we need to refer to the arrangement sketched in Fig. 3. The amplitude distribution in the second back focal plane is here obtained by a double Fourier transform, and leads to the well-known inverted "image", with magnification in the ratio of focal lengths.

The aim now is to operate in the spatial frequency spectrum plane, and to modify the image in a controlled wave plane way; in other words the frequency spectrum will be manipulated by the imposition of filters and masks. space, is that very simple changes in the spectrum plane may have, by virtue of the Fourier transform, tremendous effects on the amplitude distribution. This had already been found by $E$. Abbe in an experiment where he altered in various ways the image of a grid, by placing masks in the focal plane of the imaging lens.

As seen before, one obtains the Fourier transform of the input distribution $t_{1}\left(x_{1}, y_{1}\right)$. The purpose is to operate on $\bar{t}_{1}$ (the Fourier transform of $\left.t_{1}\right)$, by a filter operation characterized by $F\left(x_{2}, y_{2}\right)$, in order to achieve some desired result.

The second lens gives the amplitude distribution in the $\left(x_{3} y_{3}\right)$ plane:
The main reason for acting in the frequency plane, and not in the image

cies, have been designed to process raw data.

The most interesting application in this field is the use of a matched filter. Suppose a signal $s$ is to be recognized (or filtered) in a noise $n$. The amplitude distribution of the original signal can be expressed as:

$U\left(x_{1}, y_{1}\right)=s\left(x_{1}, y_{1}\right)+n\left(x_{1}, y_{1}\right)$

It can be shown that the most appropriate filter to minimize the noise relative to the wanted signal if $n$ is a random noise, is given by the expression:

$\mathrm{F}\left(x_{2}, y_{2}\right)=a(\bar{s})^{*} / \mid \tilde{n}^{\mid 2} \rightarrow=b \bar{s}^{*}$ where $(\bar{s})^{\star}$ is the complex conjugate of the Fourier transform of $s$. Calculation of (2) and (3) then gives an autocorrelation term which allows a filter to be prepared which will give optimal signal detection; in other words we have a means of performing pattern recognition.

However such calculations except in the simplest of cases are difficult and time consuming and the practical problem was to find a way of deducing $(\bar{s})^{*}$ by an alternative and simpler route. It was in 1964 that Vanderlugt suggested the basic idea for preparing such filters by semi automatic techniques using holography (Fig. 4).

Starting with the object to be picked out as the primary transducer, a suitable combination of the transformed

$U\left(x_{3}, y_{3}\right)=B \backsim t_{1}\left(x_{2}, y_{2}\right) F\left(x_{2}, y_{2}\right) \exp \left[-2 \pi i\left(x_{3} x_{2}+y_{3} y_{2}\right) / \lambda f_{2}\right] d x_{2} d y_{2}(2)$

which is the convolution of $t_{1}$ with $F$. We see immediately that besides Fourier transforms, we can perform convolutions and hence auto-correlations (convolution with complex conjugate) of two light-modulating functions of two variables.

Various types of filtering are possible which implies a wide choice in the function $F$. The simplest is the blocking filter where some spatial frequencies are removed and a well-known application is the selective raster removal from television pictures. There are, however, many other applications and very sophisticated band pass filters, conserving high or low frequen-
Fig. 3 Double lens system with modulation in the spatial frequency spectrum plane.

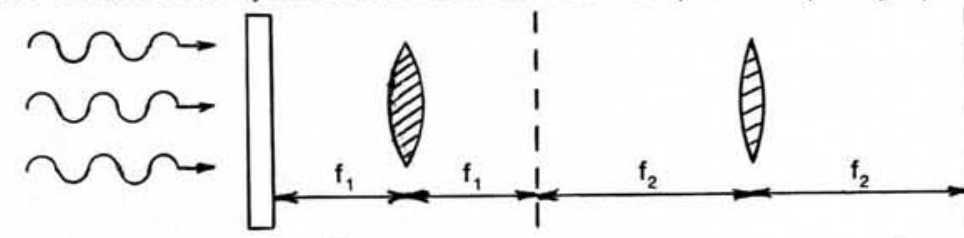

plane $\left(x_{1} y_{1}\right) \quad$ Fourier plane $\left(x_{2}, y_{2}\right)$ or spatial frequency
spectrum plane

plane $\left(x_{3} y_{3}\right)$

amplitude

object first lens focal plane second back radiation with the original radiation is made - in other words a hologram, which is the complex conjugate of the Fourier transform of the object. This then becomes the mask that is used in the analysis of the composite signal.

The Vanderlugt filter constitutes an important step in the development of optical computers, as it avoids the laborious task of calculating the required filter for a specified impulse response. In a recent paper, Pernick and De Carlo ${ }^{8}$ ) have studied the optimum imaging method for optical data processors and the applications of these optical transforms are to be found in Refs. 2, 4 and 5.

\section{Optical Computers, Type 3}

In view of the possibilities opened by the optical computers described under types 1 and 2 , it is natural to ask the question whether optical techniques can be applied to computation in the narrower meaning of the expression.

The linearity of the Fourier transform allows easy solutions for the basic operations such as addition or 

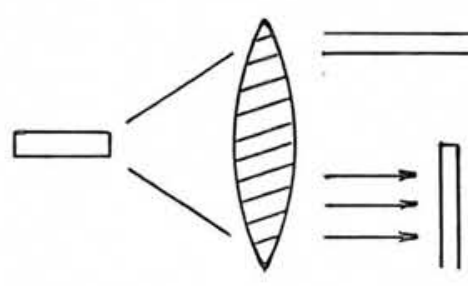

laser $s\left(x_{1}, y_{1}\right)$

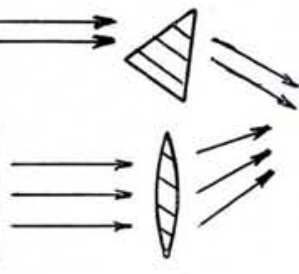

$\left.y_{1}\right)$
Fig. 4 Technique for preparing a Vanderl

ing is differentiation. To differentiate a function $f(x)$ one has to determine the filter function $F$, whose convolution with the function gives the derivative $d f / d x$.

Such a mask, called a differentiator, has an amplitude transmittance which varies linearly with distance. Higher order derivatives are obtained with the aid of transmittances varying as the power of the desired derivative. Also mixed higher order derivatives such as $\delta^{2} f / \delta x \delta y$ can be obtained, and even an integrator where the transmittance varies as the reciprocal of the distance in the transform plane. From this it follows that all the classical operators, for example the Laplacian operator or transforms, e.g. the Hilbert transform can be effected. It is important to note that the operation can be carried out in one step. As a result there is not the degradation of accuracy that occurs in electronic computing with successive processes. In the case of higher order differentiation this is particularly marked as also is the insensitivity to high slopes approaching singularities.

Finally it should be noted that besides these computing capabilities, optical devices have tremendous storage capacities, and these can be digitized. From this aspect alone, although the study of optical data processing is relatively in its infancy, one can be sure that in the computers of tomorrow, optical components will have an important place.

\section{Basic References}

1. ABBE, E., Archiv. Mikroskopische Anat. 9. (1873) 413

2. DE VELIS, J. and REYNOLDS, G., Theory and Applications of Holography (Addison-Wesley) 1967

3. PRESTON, K., Coherent Optical Computers (McGraw-Hill, New York) 1972

4. LIPSON, H.S., Optical Transforms (Academic Press) 1972

5. KOCK, W., Engineering Applications of Lasers and Holography (Plenum Press) 1975

6. GOODMAN, J., Introduction to Fourier Optics (McGraw-Hill, New York) 1968

7. GABOR, D., "Les Transformations de I'Information en Optique" Optica Acta 13 (1966) 4

8. PERNICK, B. and DE CARLO, J., "Optimum Imaging Method for Optical Data Processors" Applied Optics 16 (1977) 1

\section{Tokamak Reactors for}

\section{Break-even H. Knœpfel, Frascati}

\section{A critical study of the near-term fusion reactor programme}

Fig. 1 Schematic view of a Tokamak. The air-core transformer induces the current $I_{p}$ that heats the plasma and produces the magnetic field $B_{P}$ necessary for equilibrium and stability. The compact toroidal magnet is typical for high field $\left(B_{T} \approx 10 \mathrm{~T}\right.$ ) devices. Not shown are coils producing a vertical field $B_{V}$ for centring the plasma colum.

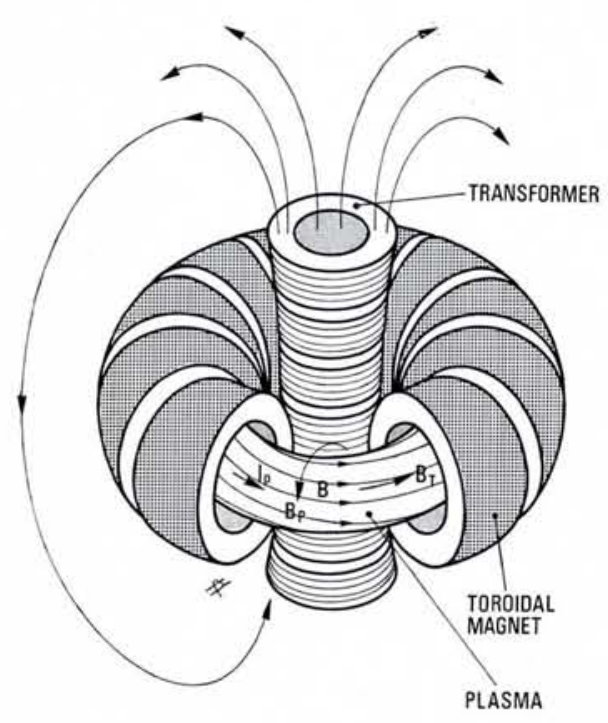

At a time when discussions on the definition of the Joint European Torus programme (JET) are still going on, it may be useful and interesting to look back for a moment to the Course held in Erice in September 1976 on Tokamak Reactors for Break even - A Critical Study of the Near Term Fusion Reactor Program. The title refers to Tokamak devices (Fig. 1) in which the thermonuclear energy produced from a deuterium-tritium plasma, represents a substantial fraction of the energy required to sustain the machine cycle and heat the plasma. The discussion at Erice was thus centred on the steps that should follow JET; implicitly, it also provided a valuable contribution to the definition of the aims and prospects of a much needed near-to-medium term European fusion programme. Such a definition could be of much help to Euratom, which is in charge of coordinating the West European fusion effort.

The present effort in the field of controlled thermonuclear fusion (Table 1) represents a substantial new experience for all those concerned with research and development pro-
(CNEN lonized Gas Laboratory)

grammes. In fusion, we find an intriguing mixture of fundamental and applied research, and of technological developments as well; moreover, the further the work proceeds, the more these $R$ \& D programmes (all equally important) will have to match the ultimate promise of fusion as a new energy source. The unusual but necessary variety of interweaved approaches and motivations is what makes fusion research to many so confusing - and, at the same time exciting; it also makes it so essentially different from other major technological and scientific adventures, such as the exploration of space, or the development of nuclear breeders,

\section{Table I: Controlled Fusion 1977}

1. Effort required to reach reactor stage may be larger than the NASA Apollo Programme.

2 Fusion has now entered into competition for funds and support with other important R \& D programmes.

3. World effort in magnetic fusion is supported by more than 3000 professionals and an estimated $700 \mathrm{M} \mathrm{\$ ;} \mathrm{more} \mathrm{than}$ $2 / 3$ are directly or indirectly for the Tokamak line.

4. There is as yet no clear indication on the scientific feasibility. 